\title{
Association of six CpG-SNPs in the inflammation-related genes with coronary heart disease
}

Xiaomin Chen ${ }^{1}$, Xiaoying Chen ${ }^{3}$, Yan Xu ${ }^{3}$, William Yang ${ }^{2}$, Nan Wu ${ }^{1,3}$, Huadan Ye $e^{3}$, Jack Y. Yang ${ }^{5}$, Qingxiao Hong ${ }^{3}$, Yanfei Xin ${ }^{4}$, Mary Qu Yang ${ }^{5}$, Youping Deng ${ }^{6,7}$ and Shiwei Duan ${ }^{3^{*}}$

\begin{abstract}
Background: Chronic inflammation has been widely considered to be the major risk factor of coronary heart disease (CHD). The goal of our study was to explore the possible association with CHD for inflammation-related single nucleotide polymorphisms (SNPs) involved in cytosine-phosphate-guanine (CpG) dinucleotides. A total of 784 CHD patients and 739 non-CHD controls were recruited from Zhejiang Province, China. Using the Sequenom MassARRAY platform, we measured the genotypes of six inflammation-related CpG-SNPs, including IL1B rs16944, IL1R2 rs2071008, PLA2G7 rs9395208, FAM5C rs12732361, CD40 rs1800686, and CD36 rs2065666). Allele and genotype frequencies were compared between CHD and non-CHD individuals using the CLUMP22 software with 10,000 Monte Carlo simulations.

Results: Allelic tests showed that PLA2G7 rs9395208 and CD40 rs1800686 were significantly associated with CHD. Moreover, IL1B rs16944, PLA2G7 rs9395208, and CD40 rs1800686 were shown to be associated with CHD under the dominant model. Further gender-based subgroup tests showed that one SNP (CD40 rs1800686) and two SNPs (FAM5C rs 12732361 and CD36 rs2065666) were associated with CHD in females and males, respectively. And the age-based subgroup tests indicated that PLA2G7 rs9395208, IL1B rs16944, and CD40 rs1800686 were associated with CHD among individuals younger than 55, younger than 65 , and over 65 , respectively.

Conclusions: In conclusion, all the six inflammation-related CpG-SNPs (rs16944, rs2071008, rs12732361, rs2065666, rs9395208, and rs1800686) were associated with CHD in the combined or subgroup tests, suggesting an important role of inflammation in the risk of CHD.
\end{abstract}

Keywords: Coronary heart disease, Inflammation, Promoter, CpG-SNP, Polymorphism

\section{Background}

Coronary heart disease (CHD) is considered to be the leading cause of mortality and morbidity in the elderly [1]. CHD has emerged as a serious burden to human health [2]. Both genetic and environmental factors were shown to play an important role in the development of CHD [3]. Environmental factors, including smoking and dietary changes, were found to be associated with CHD [4]. Heritable factors were estimated to contribute to $30-60 \%$ of the variation in the risk of CHD [3]. In addition, the incidence of CHD was generally higher in

\footnotetext{
* Correspondence: duanshiwei@nbu.edu.cn

${ }^{3}$ School of Medicine, Ningbo University, Ningbo, Zhejiang 315211, China Full list of author information is available at the end of the article
}

men than in women regardless of their menopause type [5]. Therefore, further exploration of the interactive mechanism between genes and environment is much more significant and helpful for specific diagnosis [6].

Increasing amount of evidence has indicated that inflammation is responsible for CHD [7, 8]. Many inflammation-related genes are found to be associated with risk of CHD $[9,10]$. Interleukin 1 , beta gene $(I L 1 B)$ encodes the cytokine which exerts a wide range of inflammatory activities [11]. Genetic association between IL1B and CHD has been previously found [12]. Interleukin 1 receptor, type II (IL1R2) encodes a cytokine receptor that belongs to the interleukin 1 receptor family [13]. Notably, IL1R2 is recognized as a general factor in 
inflammatory response [14]. Phospholipase A2, group VII (PLA2G7) gene encodes a kind of secreted enzyme [15], and patients with high PLA2G7 expression was found to have an increased risk of cardiovascular diseases [16]. Gene polymorphisms in family with sequence similarity 5 , member $\mathrm{C}$ (FAM5C) gene were shown to be associated with an increased risk of acute myocardial infarction [17], and elevated FAM5C levels were found in atherosclerotic plaques and coronary artery endothelium [18]. CD40 encodes a cell surface receptor that plays a pivotal role in macrophage activation and parasite immunity [19], and CD40 could up-regulate prime macrophages through a proinflammatory program in recent studies [19]. CD36 encodes a platelet receptor glycoprotein involved in different biological processes such as inflammation, atherosclerosis, and platelet activation [20]. The association between monocyte/macrophage CD36 and atherosclerosis has been found in the previous studies [21].

Single nucleotide polymorphisms (SNPs) can change biological properties of the encoded protein and affect gene expression levels in an allele-specific manner (24450106). Due to the mutability of cytosine-phosphateguanine (CpG) dinucleotides, CpG-SNPs, as an important class of cis-regulatory polymorphisms, connect genetic variation to the individual variability of the epigenome [22]. CpG-SNPs in the promoter regions had been found to be associated with multiple diseases, including type 2 diabetes [23], breast cancer [24], schizophrenia [25], and epithelial ovarian cancer [26]. In light of previous studies, we aimed to evaluate whether the six inflammation-related gene CpG-SNPs could contribute to the risk of CHD.

\section{Results and discussion}

Genotypic and allelic tests were performed for a total of six CpG-SNPs between CHD and non-CHD individuals (Table 1). According to the most recent human assembly, hg38/GRCh38, five CpG-SNPs (FAM5C rs12732361, IL1R2 rs2071008, IL1B rs16944, CD40 rs1800686, and CD36 rs2065666) were located upstream of the corresponding transcription start sites $(47,178,387,508$, and $659 \mathrm{bp}$, respectively). The remaining one (PLA2G7 rs9395208) was located in the $111 \mathrm{bp}$ downstream of transcription start sites.

As shown in Table 2, the genotype distributions of all the six SNPs in the non-CHD controls met HardyWeinberg equilibrium (HWE). Our results showed that PLA2G7 rs9395208 and CD40 rs1800686 were significantly associated with CHD on the allele level. The frequency of allele rs9395208-G was significantly lower in the case group than in the control (84.7 versus $87.3 \%$; $P=0.04, \mathrm{OR}=0.806,95 \% \mathrm{CI}=0.657-0.991)$. Meanwhile, a higher rs1800686-G frequency showed in the cases than in controls (69.4 versus $65.4 \% ; P=0.02$; OR $=1.198,95 \%$ $\mathrm{CI}=1.029-0.394)$. No significant difference was observed on the genotype level for all the CpG-SNPs $(P>0.05)$.

Gender disparities widely existed in the prevalence of CHD [27], and female CHD patients aged 40 or older have $10 \%$ greater risk of death than the males [27]. Hence, we performed a gender-based subgroup analysis

Table 1 The list of genotyping primers for six CpG-SNPs

\begin{tabular}{|c|c|c|c|}
\hline Gene & SNP & Primers & Sequence $\left(5^{\prime}\right.$ to $\left.3^{\prime}\right)$ \\
\hline \multirow[t]{3}{*}{ ILIB } & rs16944 & 1st PCR primer & ACGTTGGATGAGAGGCTCCTGCAATTGACA \\
\hline & & 2nd primer & ACGTTGGATGCTGTCTGTATTGAGGGTGTG \\
\hline & & Extend primer & GGGGTGGGTGCTGTTCTCTGCCTC \\
\hline \multirow[t]{3}{*}{ ILIR2 } & rs2071008 & 1st PCR primer & ACGTTGGATGGAAAAATCCATGCAGCCTCC \\
\hline & & 2nd primer & ACGTTGGATGTGGTGGCTGACTTTCCAAGG \\
\hline & & Extend primer & TGGGAAGAAGCAAGCACCCC \\
\hline \multirow[t]{3}{*}{ PLA2G7 } & rs9395208 & 1st PCR primer & ACGTTGGATGTGGACCCGCGGTTAACTTAG \\
\hline & & 2nd primer & ACGTTGGATGATCAGGTCTGCGGAAAGGAG \\
\hline & & Extend primer & GCATTGCCTGGCTCT \\
\hline \multirow[t]{3}{*}{ FAM5C } & rs12732361 & 1st PCR primer & ACGTTGGATGTTACACAGAGAGCCACGAAC \\
\hline & & 2nd primer & ACGTTGGATGAGGATCACCACGAATCACCC \\
\hline & & Extend primer & GAAACCCCCACCATTCCCCA \\
\hline \multirow[t]{3}{*}{ CD40 } & rs1800686 & 1st PCR primer & ACGTTGGATGATGGATGGGAAGTTGAGACG \\
\hline & & 2nd primer & ACGTTGGATGCCCAACTCAGAATTTCGCTC \\
\hline & & Extend primer & GTCGCTTTCAAAGGAAATTCCCT \\
\hline \multirow[t]{3}{*}{ CD36 } & rs2065666 & 1st PCR primer & ACGTTGGATGCTCTGAAGATATAATGACAAG \\
\hline & & 2nd primer & ACGTTGGATGCAGTTTCTCTGTTCACTTCG \\
\hline & & Extend primer & CGTTCACTTCGTTITAGTATAGAATTA \\
\hline
\end{tabular}


Table 2 Comparisons of genotype and allele frequencies between cases and controls

\begin{tabular}{|c|c|c|c|c|c|c|c|c|c|c|c|c|}
\hline \multirow{2}{*}{$\frac{S N P}{\text { IL1B rs16944 }}$} & \multirow[t]{2}{*}{ Groups } & \multicolumn{3}{|c|}{ Genotype (counts) } & \multirow[t]{2}{*}{$x^{2}$} & \multirow[t]{2}{*}{$P(d f=2)$} & \multirow[t]{2}{*}{ HWE $P$ value ${ }^{a}$} & \multicolumn{2}{|c|}{ Allele (counts) } & \multirow[t]{2}{*}{$x^{2}$} & \multirow[t]{2}{*}{$P(d f=1)$} & \multirow[t]{2}{*}{ OR $(95 \%$ Cl) } \\
\hline & & GG & $A G$ & $\mathrm{AA}$ & & & & G & A & & & \\
\hline & Cases & 220 & 380 & 176 & & & 0.627 & 820 & 732 & & & \\
\hline & Controls & 173 & 379 & 180 & 4.387 & 0.112 & 0.335 & 725 & 739 & 3.310 & 0.069 & $1.142(0.990-1.317)$ \\
\hline \multirow[t]{3}{*}{ IL1R2 rs2071008 } & & GG & GT & $\pi$ & & & & G & $\mathrm{T}$ & & & \\
\hline & Cases & 434 & 285 & 65 & & & 0.064 & 1153 & 415 & & & \\
\hline & Controls & 417 & 275 & 46 & 2.382 & 0.304 & 0.941 & 1109 & 367 & 1.020 & 0.313 & $0.919(0.781-1.082)$ \\
\hline \multirow[t]{3}{*}{ PLA2G7 rs9395208 } & & GG & GC & $\mathrm{CC}$ & & & & G & C & & & \\
\hline & Cases & 565 & 198 & 21 & & & 0.468 & 1328 & 240 & & & \\
\hline & Controls & 568 & 154 & 17 & 4.603 & 0.100 & 0.095 & 1290 & 188 & 4.210 & 0.040 & $0.806(0.657-0.991)$ \\
\hline \multirow[t]{3}{*}{ FAM5C rs 12732361} & & GG & $A G$ & $\mathrm{AA}$ & & & & G & A & & & \\
\hline & Cases & 492 & 242 & 49 & & & 0.011 & 1226 & 340 & & & \\
\hline & Controls & 431 & 254 & 52 & 3.022 & 0.221 & 0.088 & 1116 & 358 & 2.850 & 0.091 & $1.157(0.977-1.370)$ \\
\hline \multirow[t]{3}{*}{ CD40 rs1800686 } & & GG & $A G$ & $\mathrm{AA}$ & & & & G & A & & & \\
\hline & Cases & 378 & 332 & 74 & & & 0.929 & 1088 & 480 & & & \\
\hline & Controls & 317 & 333 & 89 & 5.411 & 0.067 & 0.914 & 967 & 511 & 5.440 & 0.020 & $1.198(1.029-1.394)$ \\
\hline \multirow[t]{3}{*}{ CD36 rs2065666 } & & GG & GC & CC & & & & G & C & & & \\
\hline & Cases & 439 & 288 & 56 & & & 0.356 & 1166 & 400 & & & \\
\hline & Controls & 411 & 282 & 43 & 1.24 & 0.538 & 0.555 & 1104 & 368 & 0.120 & 0.729 & $0.972(0.825-1.145)$ \\
\hline
\end{tabular}

${ }^{\mathrm{a}} H W E$ Hardy-Weinberg equilibrium; $P$ value $<0.05$ was considered a departure from HWE

to detect the difference both in genotype and allele frequencies (Table 3). It showed significant associations of FAM5C rs12732361 and CD36 rs2065666 with CHD in males on the allele level $(P=0.03, \mathrm{OR}=1.266,95 \%$ $\mathrm{CI}=1.022-1.568 ; P=0.04$, OR $=0.806,95 \% \mathrm{CI}=0.652-$ 0.996, respectively). Meanwhile, CD40 rs1800686 was shown to be significantly associated with CHD in females ( $P=0.02$, OR $=1.347,95 \% \mathrm{CI}=1.043-1.740$ by allele $)$.

Age is another well-known risk factor in the development and prognosis of CHD [28], and there were over $70 \%$ of coronary-related deaths occurred in the people older than 70 in North America and Western Europe [28]. Therefore, we further evaluated the genotype and allele frequencies in different age subgroups (Table 3). For the individuals younger than 55, the frequencies of both rs16944-G and rs2071008-G alleles were significantly lower in case group than in control group $(P=0.01$, $\mathrm{OR}=1.422,95 \% \mathrm{CI}=1.077-1.877 ; P=0.04$, $\mathrm{OR}=0.733$, $95 \% \mathrm{CI}=0.538-1.000$, respectively). For the individuals with age between 55 and 65 years old, rs16944 was associated with CHD on both genotype and allele levels $\left(\chi^{2}=6.15, P=0.04\right.$ by genotype; $P=0.03, \mathrm{OR}=1.299,95 \%$ $\mathrm{CI}=1.022-1.653$ by allele). Among the individuals older than 55, rs1800686 was also shown to be associated with CHD on both genotype and allele levels (genotype: $X^{2}=9.44, P=0.009$; allele: $P=0.003 ; \mathrm{OR}=1.456,95 \%$ $\mathrm{CI}=1.132-1.8740)$.

Additionally, we have conducted a comparison under the dominant and recessive inheritance models between cases and controls (Table 3). In the dominant model, significant associations among rs16944, rs9395208 and rs1800686 with CHD were observed. The rs16944-A and rs1800686-A alleles were risk factors for CHD (rs16944: $P=0.03, \mathrm{OR}=1.279,95 \% \mathrm{CI}=1.015-1.611$; rs1800686: $P=0.04, \mathrm{OR}=1.239,95 \% \mathrm{CI}=1.013-1.517$ ). The rs9395208-C allele was a protective factor for CHD $(P=0.03, \mathrm{OR}=0.777,95 \% \mathrm{CI}=0.616-0.979)$. Meanwhile, all the six CpG-SNPs showed no significant association with CHD in the recessive model.

Besides, a post hoc power analysis showed that our association study had strong power $(93.9-94.1 \%)$ to detect significant association of the six CpG-SNPs under an OR of 1.3.

The present study performed a comprehensive analysis of association between six CpG-SNPs (IL1B rs16944, IL1R2 rs2071008, PLA2G7 rs9395208, FAM5C rs12732361, CD40 rs1800686, and CD36 rs2065666) and CHD. At the allelic level, $C D 40$ rs1800686-G was found to be a risk factor, while PLA2G7 rs9395208-G was found to be a protective factor. Moreover, all the CpG-SNPs were significantly associated with the risk of $\mathrm{CHD}$ in the combined or subgroup analyses.

Inflammation represents an important feature in the process of atherosclerosis, which can form, destabilize, and rupture atherosclerotic plaques, finally causing CHD [29]. Over the years, researchers have found some inflammatory factors related to the pathogenesis and prognosis of CHD, such as CLOCK SNP rs4580704 [30], ICAM-1 
Table 3 A list of SNPs associated with CHD in the subgroup tests

\begin{tabular}{|c|c|c|c|c|c|c|c|}
\hline Gene & SNP & Model or subgroup & Group & Genotype or allele (counts) & $x^{2}$ & $P(d f)$ & OR $(95 \%$ Cl) \\
\hline \multirow[t]{2}{*}{ ILIB } & rs16944 & Dominant & Cases & GG/GA + AA (220/556) & 4.350 & $0.037(1)$ & $1.279(1.015-1.611)$ \\
\hline & & & Controls & GG/GA + AA (173/559) & & & \\
\hline \multirow[t]{2}{*}{ ILIB } & rs16944 & $\leq 55$ years of age & Cases & G/A (236/236) & 6.190 & $0.013(1)$ & $1.422(1.077-1.877)$ \\
\hline & & & Controls & G/A (292/248) & & & \\
\hline \multirow[t]{2}{*}{ ILIB } & rs16944 & $55-65$ years of age & Cases & GG/GA/AA (79/134/57) & 6.147 & $0.046(2)$ & \\
\hline & & & Controls & GG/GA/AA (53/145/66) & & & \\
\hline \multirow[t]{2}{*}{ ILIB } & rs16944 & $55-65$ years of age & Cases & G/A (292/248) & 4.560 & $0.033(1)$ & $1.299(1.022-1.653)$ \\
\hline & & & Cases & G/A (251/277) & & & \\
\hline \multirow[t]{2}{*}{ ILIR2 } & rs2071008 & $\leq 55$ years of age & Controls & G/T (254/106) & 3.860 & $0.049(1)$ & $0.733(0.538-1.000)$ \\
\hline & & & Cases & G/T (366/112) & & & \\
\hline \multirow[t]{2}{*}{ PLA2G7 } & rs9395208 & Dominant & Cases & GG/GC+ CC (565/219) & 4.590 & $0.032(1)$ & $0.777(0.616-0.979)$ \\
\hline & & & Controls & GG/GC+ CC (568/171) & & & \\
\hline \multirow[t]{2}{*}{ PLA2G7 } & rs9395208 & $\leq 55$ years of age & Cases & G/C (295/65) & 5.750 & $0.016(1)$ & $0.627(0.427-0.920)$ \\
\hline & & & Controls & G/C (420/58) & & & \\
\hline \multirow[t]{2}{*}{ FAM5C } & rs12732361 & Male & Cases & G/C (849/225) & 4.660 & $0.031(1)$ & $1.266(1.022-1.568)$ \\
\hline & & & Controls & G/C (629/211) & & & \\
\hline \multirow[t]{2}{*}{ CD40 } & rs1800686 & Female & Cases & G/A (354/138) & 5.230 & $0.022(1)$ & $1.347(1.043-1.740)$ \\
\hline & & & Controls & G/A (417/219) & & & \\
\hline \multirow[t]{2}{*}{ CD40 } & rs1800686 & Dominant & Cases & GG/GA + AA (378/406) & 4.340 & $0.037(1)$ & $1.239(1.013-1.517)$ \\
\hline & & & Controls & GG/GA + AA (317/422) & & & \\
\hline \multirow[t]{2}{*}{ CD40 } & rs1800686 & $\geq 65$ years of age & Cases & GG/GA/AA (163/146/24) & 9.437 & $0.009(2)$ & \\
\hline & & & Controls & GG/GA/AA (90/109/32) & & & \\
\hline \multirow[t]{2}{*}{ CD40 } & rs1800686 & $\geq 65$ years of age & Cases & G/A (472/194) & 8.600 & $0.0034(1)$ & $1.456(1.132-1.874)$ \\
\hline & & & Controls & G/A (289/173) & & & \\
\hline \multirow[t]{2}{*}{ CD36 } & rs2065666 & Male & Cases & G/C (790/286) & 4.000 & $0.046(1)$ & $0.806(0.652-0.996)$ \\
\hline & & & Controls & G/C (641/187) & & & \\
\hline
\end{tabular}

SNP rs281432 [31], and NFKB1 SNP rs28362491 [32]. Some of these inflammation-related genes were expected to be drug targets for the control and treatment of CHD. Meanwhile, we hypothesized that hereditable methylation could be associated with CHD. Because of low minor allele frequency or weak haplotype associations, genomewide searches for genetic risk factors for $\mathrm{CHD}$ have in general not investigated the CpG-SNPs. Our previous work [33] indicated that CpG-SNPs of the thrombotic pathway genes contributed to the risk of $\mathrm{CHD}$, which suggested a clue for investigating the contributions of the inflammation-related CpG-SNPs to the susceptibility to $\mathrm{CHD}$.

PLA2G7 gene encodes a secreted enzyme whose activity is associated with CHD [34]. PLA2G7 functions as a biomarker of plaque inflammation and stability [35]. Several PLA2G7 SNPs (e.g., rs7756935, rs1805017, and rs13210554) have been reported on the susceptibility to CHD [35, 36]. Here, we discovered a significant association of PLA2G7 CpG-SNP rs9395208 with CHD, even in the individuals aged $\leq 55$, providing additional evidence of age dimorphism in the risk of CHD. Additionally, Jiang et al. [15] have reported that the correlation between PLA2G7 methylation and CHD risk in females is independent of age, smoking, diabetes, and hypertension, which indicated a close relationship between PLA2G7 and hereditable methylation in the pathogenic mechanism of CHD.

The contribution of $C D 40$ rs1800686 is another main finding in the current study. $C D 40$ is considered to determine $\mathrm{T}$ cell responses to antigen presentation and $\mathrm{B}$ cells immunoglobulin isotype switching, which plays a key role in the inflammatory and prothrombotic processes by bonding with CD40 ligand (CD40L) in atherosclerosis [37]. Previous study provided evidence of association of CD40 rs1883832 with an overall increased risk of CHD in Chinese population [38]. In the current study, a similar result was drawn that $C D 40$ rs1800686-A allele contributed 
to $\mathrm{CHD}$ risk. Age and gender are considered as two independent risk factors in CHD [28, 39]. Subsequent subgroup analysis identified age and gender differences existed in the rs1800686, which revealed an age- and gender-based mechanism on the genetic variations.

As a scavenger receptor, CD36 is not only involved in the metabolism of lipids but also plays an important role in the adhesion of negatively charged macromolecules [40]. CD36 is widely expressed in cells and tissues including microvascular endothelial cells, monocytes, and macrophages [41]. Previous study implied that increased CD36 expression level could reflect the severity of coronary artery atherosclerosis [42]. Several CD36 SNPs (e.g., rs5956, rs3173798, and rs3211892) have been reported to be associated with CHD [42]. Here, we detected a significant protection role of CD36 CpG-SNP rs2065666 $\mathrm{G}$ carries in CHD male patients. Our data suggested a possible molecular mechanism through which SNP could influence a phenotype.

Interleukins (IL-1B and IL1R2) were shown to play a role in the inflammatory response, and they lead to the development of atherosclerotic plaques [43, 44]. Recently, gender dimorphism was observed in the association of $I L-1 B$ polymorphism with CHD [45]. We find no significant difference on $I L-1 B$ rs16944 allele or genotype level by gender subgroup analysis. However, agebased subgroup tests showed that $I L-1 B$ rs16944-G was a risk factor of CHD in younger population, while IL1R2 rs2071008-G was a protective factor. Further validation study or functional analysis of these variants is needed in the future.

Increased expression of FAM5C may be induced by inflammatory stimuli [17]. This case-control study observed significant associations between FAM5C rs12732361 polymorphisms and CHD only in males. Due to a lack of CHD study on FAM 5 C, more investigations are needed to validate our results.

There are also limitations in the current study. The findings of our study are limited to a small population with documented CHD and cannot be generalized to the population at large. Although we found positive results in the current study, the power effect might be reduced by strict multiple adjustment or subgroup analyses. Therefore, larger sample size and other ethnic populations are required to be investigated.

\section{Conclusions}

In conclusion, our case-control study suggested that the six inflammation-related CpG-SNPs were significantly associated with the risk of CHD in the combined or the subgroup samples. Moreover, our results also revealed that IL1B rs16944, PLA2G7 rs9395208, and CD40 rs1800686 had a significant contribution to the risk of CHD under the dominant model.

\section{Methods}

\section{Sample collection}

A total of $784 \mathrm{CHD}$ patients and 739 healthy controls were collected from Ningbo First Hospital of Ningbo University between May 2008 and April 2015 in Zhejiang province, China. CHD patients and non-CHD controls were defined as described before [33, 46]. Patients with congenital heart disease, cardiomyopathy, liver disease, and renal disease were excluded. Blood samples were collected in $3.2 \%$ citrate sodium-treated tubes and processed in the central clinical laboratory of the hospital. All the participants provided written informed consent form under a protocol approved by the Medical Ethics Committees of Ningbo First Hospital and Ningbo University.

\section{SNP selection and genotyping}

The selected CpG-SNPs are on the promoter of inflammation-related genes. Meanwhile, the minor allele frequencies of the selected CpG-SNPs are over $10 \%$ in HapMap HCB population. CpG-SNPs with design problems or failed assays were excluded. Finally, six CpG-SNPs in inflammation-related genes were included in the current study. DNA extraction and quantification were described as previously $[33,46]$. The polymerase chain reaction (PCR) amplification was performed on the GeneAmp PCR System 9700 (Applied Biosystems, Foster City, CA, USA), and the genotyping was performed on the MassARRAY iPLEX ${ }^{\circ}$ assay platform (Sequenom, San Diego, CA, USA). The sequences of the amplification and extension primers for the six CpG-SNPs were shown in Table 1.

\section{Statistical analyses}

Genotype and allele frequencies of the polymorphisms between cases and controls were calculated by the CLUMP22 software with 10,000 Monte Carlo simulations [47]. The distribution of Hardy-Weinberg equilibrium (HWE) was tested using the Arlequin program (version 3.5, Bern, Switzerland), and $P>0.05$ was considered to be in HWE. Power analysis was performed using the Power and Sample Size Calculation software (v3.0.43). A twotailed $P<0.05$ was considered statistically significant.

\section{Competing interests \\ The authors declare that they have no competing interests.}

\section{Authors' contributions}

$X M C$, SWD, and MQY conceived and designed the experiments. XYC, YX, NW, HDY, QXH, and YPD performed the experiments. XYC, HDY, and WY analyzed the data. JYY and YFX contributed reagents/materials/analysis tools. $X Y C$ and $Y X$ wrote the paper. $X M C, X Y C$, and $Y X$ contributed equally to this work. All authors read and approved the final manuscript.

\section{Declarations}

The research and publication was supported by the grants from the National Natural Science Foundation of China (31100919, 81371469), Zhejiang Provincial Natural Science Foundation (LR13H020003), Ningbo City Medical Science and Technology projects (2014A20), and K. C. Wong Magna Fund in Ningbo University. This article has been published as part of Human Genomics volume 10 Supplement 
2, 2016: from genes to systems genomics: human genomics. The full contents of the supplement are available online at http://humgenomics.biomedcentral.com/articles/ supplements/volume-10-supplement-2.

\section{Author details}

${ }^{1}$ Cardiovascular Center of Ningbo First Hospital, Ningbo University, Ningbo, Zhejiang 315010, China. ${ }^{2}$ Texas Advanced Computing Center, University of Texas at Austin, 10100 Burnet Road (R8700), Austin, TX 78758-4497, USA. ${ }^{3}$ School of Medicine, Ningbo University, Ningbo, Zhejiang 315211, China. ${ }^{4}$ Center of Safety Evaluation, Zhejiang Academy of Medical Sciences, Hangzhou, Zhejiang 310007, China. 'MidSouth Bioinformatics Center, Department of Information Science, George Washington Donaghey College of Engineering and Information Science, and Joint Bioinformatics Graduate Program, University of Arkansas at Little Rock and University of Arkansas for Medical Sciences, 2881 S. University Ave, Little Rock, AR 72204, USA. ${ }^{6}$ Medical College, Wuhan University of Science and Technology, Wuhan 430064, China. ${ }^{7}$ Department of Internal Medicine and Biochemistry, Rush University Medical Center, Chicago, IL 60612, USA.

\section{Published: 25 July 2016}

\section{References}

1. Lauer MS. Advancing cardiovascular research. Chest. 2012;141:500-5

2. Beatty $A L$, Ku IA, Bibbins-Domingo K, Christenson RH, DeFilippi CR, Ganz $P$, et al. Traditional risk factors versus biomarkers for prediction of secondary events in patients with stable coronary heart disease: from the heart and soul study. J Am Heart Assoc. 2015;4. doi:10.1161/JAHA.114.001646.

3. Mega JL, Stitziel NO, Smith JG, Chasman DI, Caulfield MJ, Devlin JJ, et al. Genetic risk, coronary heart disease events, and the clinical benefit of statin therapy: an analysis of primary and secondary prevention trials. Lancet. 2015;385:2264-71.

4. Dalen JE, Alpert JS, Goldberg RJ, Weinstein RS. The epidemic of the 20(th) century: coronary heart disease. Am J Med. 2014;127:807-12.

5. Kim C, Cushman M, Khodneva Y, Lisabeth LD, Judd S, Kleindorfer DO, et al. Risk of incident coronary heart disease events in men compared to women by menopause type and race. J Am Heart Assoc. 2015;4. doi:10.1161/JAHA.115.001881.

6. Xiaoying C, Huadan Y, Qingxiao H, Annan Z, Linlin T, Shiwei D. The effects of DNA methylation on the homeostasis in vascular diseases. Yi Chuan. 2015;37:221-32

7. Ross R. Atherosclerosis_an inflammatory disease. N Engl J Med. 1999;340:115-26.

8. Rothenbacher D, Kleiner A, Koenig W, Primatesta P, Breitling LP, Brenner H. Relationship between inflammatory cytokines and uric acid levels with adverse cardiovascular outcomes in patients with stable coronary heart disease. PLoS One. 2012;7:e45907.

9. Gigante B, Strawbridge RJ, Velasquez IM, Golabkesh Z, Silveira A, Goel A, et al. Analysis of the role of interleukin 6 receptor haplotypes in the regulation of circulating levels of inflammatory biomarkers and risk of coronary heart disease. PLoS One. 2015;10:e0119980.

10. Li L, He M, Zhou L, Miao X, Wu F, Huang S, et al. A solute carrier family 22 member 3 variant $r 3088442 \mathrm{G} \rightarrow \mathrm{A}$ associated with coronary heart disease inhibits lipopolysaccharide-induced inflammatory response. J Biol Chem. 2015;290:5328-40.

11. Stahelova A, Mrazek F, Smizansky M, Petrek M, Gallo J. Variation in the IL1B, TNF and IL6 genes and individual susceptibility to prosthetic joint infection. BMC Immunol. 2012;13:25.

12. Zhang YM, Zhong LJ, He BX, Li WC, Nie J, Wang $X$, et al. The correlation between polymorphism at position $-511 \mathrm{C} / \mathrm{T}$ in the promoter region of interleukin $1 \mathrm{~B}$ and the severity of coronary heart disease. Zhonghua Yi Xue Yi Chuan Xue Za Zhi. 2006;23:86-8.

13. Palomo J, Dietrich D, Martin P, Palmer G, Gabay C. The interleukin (IL)-1 cytokine family - balance between agonists and antagonists in inflammatory diseases. Cytokine. 2015;76:25-37.

14. Garlanda C, Riva F, Bonavita E, Mantovani A. Negative regulatory receptors of the IL-1 family. Semin Immunol. 2013;25:408-15.

15. Jiang $D$, Zheng D, Wang L, Huang $Y$, Liu H, Xu L, et al. Elevated PLA2G7 gene promoter methylation as a gender-specific marker of aging increases the risk of coronary heart disease in females. PLoS One. 2013;8:e59752.

16. Vainio P, Lehtinen L, Mirtti T, Hilvo M, Seppanen-Laakso T, Virtanen J, et al. Phospholipase PLA2G7, associated with aggressive prostate cancer, promotes prostate cancer cell migration and invasion and is inhibited by statins. Oncotarget. 2011;2:1176-90.

17. Sato J, Kinugasa M, Satomi-Kobayashi S, Hatakeyama K, Knox AJ, Asada Y, et al. Family with sequence similarity 5 , member C (FAM5C) increases leukocyte adhesion molecules in vascular endothelial cells: implication in vascular inflammation. PLoS One. 2014;9:e107236.

18. Kim CW, Song H, Kumar S, Nam D, Kwon HS, Chang KH, et al. Antiinflammatory and antiatherogenic role of BMP receptor II in endothelial cells. Arterioscler Thromb Vasc Biol. 2013;33:1350-9.

19. Morgado P, Sudarshana DM, Gov L, Harker KS, Lam T, Casali P, et al. Type II toxoplasma gondii induction of CD40 on infected macrophages enhances interleukin-12 responses. Infect Immun. 2014;82:4047-55.

20. Petta S, Handberg A, Marchesini G, Camma C, Di Marco V, Cabibi D, et al. High sCD36 plasma level is associated with steatosis and its severity in patients with genotype 1 chronic hepatitis C. J Viral Hepat. 2013;20:174-82.

21. Collot-Teixeira S, Martin J, McDermott-Roe C, Poston R, McGregor JL. CD36 and macrophages in atherosclerosis. Cardiovasc Res. 2007;75:468-77.

22. Shoemaker R, Deng J, Wang W, Zhang K. Allele-specific methylation is prevalent and is contributed by CpG-SNPs in the human genome. Genome Res. 2010;20:883-9.

23. Dayeh TA, Olsson AH, Volkov $P$, Almgren $P$, Ronn $T$, Ling C. Identification of CpG-SNPs associated with type 2 diabetes and differential DNA methylation in human pancreatic islets. Diabetologia. 2013;56:1036-46.

24. Harlid S, Ivarsson MI, Butt S, Hussain S, Grzybowska E, Eyfjord JE, et al. A candidate CpG SNP approach identifies a breast cancer associated ESR1SNP. Int J Cancer. 2011;129:1689-98.

25. Bani-Fatemi A, Goncalves VF, Zai C, de Souza R, Le Foll B, Kennedy JL, et al. Analysis of CpG SNPs in 34 genes: association test with suicide attempt in schizophrenia. Schizophr Res. 2013;147:262-8.

26. Koestler DC, Chalise P, Cicek MS, Cunningham JM, Armasu S, Larson MC, et al. Integrative genomic analysis identifies epigenetic marks that mediate genetic risk for epithelial ovarian cancer. BMC Med Genomics. 2014;7:8.

27. Cline JL, Beckie TM. The relationships between FAM5C SNP (rs10920501) variability and metabolic syndrome and inflammation in women with coronary heart disease. Biol Res Nurs. 2013;15:160-6.

28. Che JJ, Shao YX, Li GP. Association between rs1049673 polymorphism in CD36 and premature coronary heart disease. Genet Mol Res. 2014;13:7708-17.

29. Hoffmeister A, Rothenbacher D, Bazner U, Frohlich M, Brenner H, Hombach $V$, et al. Role of novel markers of inflammation in patients with stable coronary heart disease. Am J Cardiol. 2001;87:262-6.

30. Gomez-Delgado F, Garcia-Rios A, Alcala-Diaz JF, Rangel-Zuniga O, DelgadoLista J, Yubero-Serrano EM, et al. Chronic consumption of a low-fat diet improves cardiometabolic risk factors according to the CLOCK gene in patients with coronary heart disease. Mol Nutr Food Res. 2015;59:2556-64.

31. Chou CH, Ueng KC, Liu YF, Wu CH, Yang SF, Wang PH. Impact of intercellular adhesion molecule-1 genetic polymorphisms on coronary artery disease susceptibility in Taiwanese subjects. Int J Med Sci. 2015;12:510-6.

32. Lai HM, Li XM, Yang YN, Ma YT, Xu R, Pan S, et al. Genetic variation in NFKB1 and NFKBIA and susceptibility to coronary artery disease in a Chinese Uygur population. PLoS One. 2015;10:e0129144.

33. Ye H, Zhou A, Hong Q, Chen X, Xin Y, Tang $L$, et al. Association of seven thrombotic pathway gene CpG-SNPs with coronary heart disease. Biomed Pharmacother. 2015;72:98-102.

34. Vittos O, Toana B, Vittos A, Moldoveanu E. Lipoprotein-associated phospholipase A2 (Lp-PLA2): a review of its role and significance as a cardiovascular biomarker. Biomarkers. 2012;17:289-302.

35. Xu L, Zhou J, Huang S, Huang Y, Le Y, Jiang D, et al. An association study between genetic polymorphisms related to lipoprotein-associated phospholipase A(2) and coronary heart disease. Exp Ther Med. 2013;5:742-50.

36. Hou L, Chen S, Yu H, Lu X, Chen J, Wang L, et al. Associations of PLA2G7 gene polymorphisms with plasma lipoprotein-associated phospholipase A2 activity and coronary heart disease in a Chinese Han population: the Beijing atherosclerosis study. Hum Genet. 2009;125:11-20.

37. Blanco-Colio LM, Martin-Ventura JL, de Teresa E, Farsang C, Gaw A, Gensini $\mathrm{G}$, et al. Atorvastatin decreases elevated soluble CD40L in subjects at high cardiovascular risk. Atorvastatin on inflammatory markers study: a substudy of ACTFAST. Kidney Int Suppl. 2008:S60-3. doi:10.1038/ki.2008.514.

38. Yun Y, Ma C, Ma X. The SNP rs 1883832 in CD40 gene and risk of atherosclerosis in Chinese population: a meta-analysis. PLoS One. 2014;9:e97289.

39. European Heart Rhythm A, European Association for Cardio-Thoracic S, Camm AJ, Kirchhof P, Lip GY, Schotten U, et al. Guidelines for the 
management of atrial fibrillation: the Task Force for the Management of Atrial Fibrillation of the European Society of Cardiology (ESC). Europace. 2010;12:1360-420.

40. Zhang S, Wang W, Li J, Cheng K, Zhou J, Zhu D, et al. Behavioral characterization of CD36 knockout mice with SHIRPA primary screen. Behav Brain Res. 2015;299:90-6.

41. Ramos-Arellano LE, Munoz-Valle JF, De la Cruz-Mosso U, Salgado-Bernabe AB, Castro-Alarcon N, Parra-Rojas I. Circulating CD36 and oxLDL levels are associated with cardiovascular risk factors in young subjects. BMC Cardiovasc Disord. 2014;14:54.

42. Zhang Y, Ling ZY, Deng SB, Du HA, Yin YH, Yuan J, et al. Associations between CD36 gene polymorphisms and susceptibility to coronary artery heart disease. Braz J Med Biol Res. 2014;47:895-903.

43. He F, Teng X, Gu H, Liu H, Zhou Z, Zhao Y, et al. Interleukin-6 receptor rs $7529229 \mathrm{~T} / \mathrm{C}$ polymorphism is associated with left main coronary artery disease phenotype in a Chinese population. Int J Mol Sci. 2014;15:5623-33.

44. McGeachie M, Ramoni RL, Mychaleckyj JC, Furie KL, Dreyfuss JM, Liu Y, et al. Integrative predictive model of coronary artery calcification in atherosclerosis. Circulation. 2009;120:2448-54.

45. Qi X, Bai F, You C, Guo X, Shi L, Hu Z. High-resolution melting technology for detecting genetic polymorphisms of IL-1beta and IL-1Ra in patients with coronary artery disease. Xi Bao Yu Fen Zi Mian Yi Xue Za Zhi. 2015:31:816-20.

46. Xu L, Chen X, Ye H, Hong Q, Xu M, Duan S. Association of four CpG-SNPs in the vascular-related genes with coronary heart disease. Biomed Pharmacother. 2015;70:80-3.

47. Huang Y, Zhou J, Ye H, Xu L, Le Y, Yang X, et al. Relationship between chemokine (C-X-C motif) ligand 12 gene variant (rs1746048) and coronary heart disease: case-control study and meta-analysis. Gene. 2013;521:38-44.

\section{Submit your next manuscript to BioMed Central and we will help you at every step:}

- We accept pre-submission inquiries

- Our selector tool helps you to find the most relevant journal

- We provide round the clock customer support

- Convenient online submission

- Thorough peer review

- Inclusion in PubMed and all major indexing services

- Maximum visibility for your research

Submit your manuscript at www.biomedcentral.com/submit

C Biomed Central 